\title{
THE CONNECTEDNESS OF SYMMETRIC AND SKEW-SYMMETRIC DEGENERACY LOCI: EVEN RANKS
}

\author{
LORING W. TU
}

\begin{abstract}
A degeneracy locus is the set of points where a vector-bundle map has rank at most a given integer. Such a set is symmetric or skew-symmetric according as whether the vector-bundle map is symmetric or skew-symmetric. We prove a connectedness result, first conjectured by Fulton and Lazarsfeld, for skew-symmetric degeneracy loci and for symmetric degeneracy loci of even ranks.
\end{abstract}

\section{INTRODUCTION}

In this paper we prove a conjecture of Fulton and Lazarsfeld on the connectedness of symmetric and skew-symmetric degeneracy loci, when the rank is even. Since skew-symmetric maps always have even ranks, this settles the conjecture in the skew-symmetric case. The odd-rank symmetric case remains open.

Let $E$ and $F$ be complex vector bundles over an irreducible variety $X$, and $u: E \rightarrow F$ a vector-bundle morphism between them. For a given nonnegative integer $r$, the degeneracy locus of rank $r$ of $u$ is defined to be

$$
D_{r}(u)=\{x \in X \mid \operatorname{rank} u(x) \leq r\} .
$$

The concept of a degeneracy locus is quite general, and includes as special cases all projective hypersurfaces, complete intersections, zero sets of sections of a vector bundle, and dependency loci of sections. However, in algebraic geometry one often encounters not only general vector-bundle morphisms, but morphisms satisfying symmetry conditions. If $L$ is a line bundle over $X$, a morphism $u: E \otimes E \rightarrow L$ is said to be symmetric if it is symmetric on each fiber; similarly for a skew-symmetric morphism. Their degeneracy loci are defined as in (0.1). As examples of symmetric bundle morphisms we cite (1) the second fundamental form of a smooth hypersurface in a projective space, and (2) the differential of the period map of a family of varieties. For more background information on degeneracy loci the reader is referred to [T].

Received by the editors January 4, 1988.

1980 Mathematics Subject Classification (1985 Revision). Primary 14M12; Secondary 14C17, $14 \mathrm{~F} 05$.

Key words and phrases. Connectedness, determinantal varieties, degeneracy loci, symmetric degeneracy loci, skew-symmetric degeneracy loci, ample vector bundles, isotropic subspaces, rank. 
These three types of vector-bundle morphisms may be viewed as sections of the bundles $\operatorname{Hom}(E, F),\left(\operatorname{Sym}^{2} E^{*}\right) \otimes L$, and $\left(\bigwedge^{2} E^{*}\right) \otimes L$ respectively, where $E^{*}$ denotes the dual of $E$. In [FL1] Fulton and Lazarsfeld proved that if $\operatorname{Hom}(E, F)$ is ample and $u$ is a section of $\operatorname{Hom}(E, F)$, then $D_{r}(u)$ is connected provided its expected dimension, $\operatorname{dim} X-(e-r)(f-r)$, is at least one. They conjectured the following statements for symmetric and skew-symmetric degeneracy loci [FL2, Remark (2), p. 50].

Conjecture 0.2. Let $E$ be a vector bundle of rank $e$ and $L$ a line bundle over the irreducible variety $X$. Suppose $u: E \otimes E \rightarrow L$ is a symmetric bundle map and $r$ a nonnegative integer. If $\left(\mathrm{Sym}^{2} E^{*}\right) \otimes L$ is ample and $\operatorname{dim} X-\left(\begin{array}{c}e-r+1 \\ 2\end{array}\right) \geq 1$, then $D_{r}(u)$ is connected.

Conjecture 0.3. Suppose $u$ : $E \otimes E \rightarrow L$ is a skew-symmetric bundle map and $r$ a nonnegative even integer. If $\left(\bigwedge^{2} E^{*}\right) \otimes L$ is ample and $\operatorname{dim} X-\left(\begin{array}{c}e-r \\ 2\end{array}\right) \geq 1$, then $D_{r}(u)$ is connected.

We prove both conjectures for even values of $r$, thus settling Conjecture 0.3 completely. The strategy of the proof is to represent a symmetric degeneracy locus of even rank as the image of a zero locus on a Grassmann bundle, as in Pragacz [P], using the characterization of the rank of a symmetric matrix by the dimensions of its isotropic subspaces. It then suffices to prove the connectedness of the zero locus. If the zero locus were that of a section of an ample vector bundle, its connectedness would follow immediately from the well-known Lefschetz-type theorem for ample vector bundles [S]. Unfortunately, the relevant vector bundle on the Grassmann bundle is no longer ample, and so we try to prove the connectedness by computing cohomology groups. This is done using a construction by which the cohomology of the complement of the zero locus is related to the cohomology of an affine variety. One hitch here is that the crucial cohomology comparison lemma of Fulton and Lazarsfeld [FL1, Lemma 1.6] applies only to birational maps with very restrictive fiber dimensions and is not applicable to our situation. We formulate and prove a generalization (Lemma 3.6) of the Fulton-Lazarsfeld lemma that compares the cohomology of a variety $M$ with that of an affine image of $M$, allowing arbitrary positive-dimensional fibers and thereby completing the proof.

The author is grateful to Ron Donagi for helpful discussions, and to Rick Miranda whose Mountain West Algebraic Geometry Workshop provided a forum for this work and whose comments greatly simplified the exposition of this paper.

Notational convention. Throughout this paper, by $\operatorname{dim} X$ we mean the complex dimension of $X$.

\section{ISOTROPIC SUBSPACES}

In this section we establish the characterization of the rank of a symmetric map by the dimensions of its isotropic subspaces. The result is undoubtedly 
well-known to the experts and its inclusion is justified only by the lack of a suitable reference. So let $E$ be a complex vector space of dimension $e$, and let $E^{*}$ be its dual $\operatorname{Hom}(E, \mathbf{C})$. A linear map $f: E \rightarrow E^{*}$ is said to be symmetric if $f=f^{*}$, where $f^{*}: E \rightarrow E^{*}$ is the dual of $f$. Equivalently, a linear map $f: E \rightarrow E^{*}$ is symmetric if and only if its associated bilinear form $\phi_{f}: E \times E \rightarrow$ $\mathrm{C}$ is symmetric. Since there is a bijection between the set of all symmetric maps $f: E \rightarrow E^{*}$ and the set of all symmetric bilinear forms $\phi: E \times E \rightarrow \mathbf{C}$, we will speak interchangeably of the two sets. For example, we define the kernel of a symmetric bilinear form to be the kernel of its associated linear map. The following characterization of the rank of a linear map is a standard fact from linear algebra.

Proposition 1.1. A linear map $f: E \rightarrow E^{*}$ has rank $\leq r$ if and only if $\operatorname{dim}(\operatorname{ker} f)$ $\geq e-r$, where $e=\operatorname{dim} E$.

Recall that an isotropic subspace of a symmetric bilinear form $\phi: E \times E \rightarrow \mathbf{C}$ is a subspace $V$ of $E$ such that $\left.\phi\right|_{V}: V \times V \rightarrow \mathbf{C}$ is identically zero. For a symmetric map $f: E \rightarrow E^{*}$ there is a more interesting characterization of the rank in terms of isotropic subspaces (Proposition 1.4).

Proposition 1.2. Let $\phi: E \times E \rightarrow \mathbf{C}$ be a symmetric bilinear form on a vector space $E$ of dimension $e$. Then the dimension of any isotropic subspace of $\phi$ is at most $e-((\operatorname{rank} \phi) / 2)$.

Proof. Denote by $K$ the kernel of the associated linear map $f: E \rightarrow E^{*}$ and let $V$ be a maximal isotropic subspace. Clearly, by the symmetry of $f, K \subset V$ and $\phi$ induces a bilinear map $\phi^{\prime}:(V / K) \times(E / V) \rightarrow \mathbf{C}$. By the definition of $K$, the associated linear map $V / K \rightarrow(E / V)^{*}$ of $\phi^{\prime}$ is injective. Therefore,

$$
\operatorname{dim} V-\operatorname{dim} K \leq e-\operatorname{dim} V .
$$

Since $\operatorname{dim} K=e-\operatorname{rank} \phi$, the proposition follows.

A symmetric bilinear map $\phi: E \times E \rightarrow \mathbf{C}$ is said to be nondegenerate if $\phi(x, y)=0$ for all $y$ in $E$ implies that $x=0$.

Proposition 1.3. Let $\phi: E \times E \rightarrow \mathbf{C}$ be a symmetric bilinear form on a vector space $E$ of dimension $e$. Then

(i) All maximal isotropic subspaces of $\phi$ have the same dimension.

(ii) If rank $\phi$ is even, say $2 p$, then a maximal isotropic subspace of $\phi$ has dimension $e-p$.

(iii) If rank $\phi$ is odd, say $2 p+1$, then a maximal isotropic subspace of $\phi$ has dimension $e-p-1$.

Proof. (i) Denote by $K$ the kernel of $\phi$. Then $\phi$ induces a nondegenerate symmetric bilinear map $\phi^{\prime}: E / K \times E / K \rightarrow \mathbf{C}$. Any maximal isotropic subspace $V$ of $\phi$ must contain $K$, and $V / K$ is a maximal isotropic subspace of $\phi^{\prime}$. By [L, Corollary 2, p. 362] all maximal isotropic subspaces of the nondegenerate $\phi^{\prime}$ have the same dimension. So if $V_{1}$ and $V_{2}$ are two maximal isotropic 
subspaces of $\phi$, then $\operatorname{dim} V_{1} / K=\operatorname{dim} V_{2} / K$. It follows that $V_{1}$ and $V_{2}$ have the same dimension.

(ii) Suppose rank $\phi=2 p$. Relative to some basis of $E$, the bilinear form $\phi$ is represented by the matrix

$$
\left[\begin{array}{lll}
0_{p} & I_{p} & 0 \\
I_{p} & 0_{p} & 0 \\
0 & 0 & 0_{e-2 p}
\end{array}\right],
$$

where $I_{p}$ is the $p \times p$ identity matrix and $0_{p}$ is the $p \times p$ zero matrix. This shows that $\phi$ has an isotropic subspace of dimension $e-p$, which is the maximum possible by Proposition 1.2.

(iii) Suppose $\operatorname{rank} \phi=2 p+1$. Relative to some basis of $E$, the bilinear form $\phi$ is represented by the matrix

$$
\left[\begin{array}{llll}
1 & & & \\
& 0_{p} & I_{p} & \\
& I_{p} & 0_{p} & \\
& & & 0_{e-2 p-1}
\end{array}\right],
$$

which shows that $\phi$ has an isotropic subspace of dimension $e-p-1$, the maximum possible by Proposition 1.2.

Using this proposition we can construct a table of the dimensions of the maximal isotropic subspaces $V$ of a symmetric bilinear map $\phi: E \times E \rightarrow \mathbf{C}$.

$$
\begin{array}{ccccccccccc}
\operatorname{rank} \phi & 0 & 1 & 2 & 3 & 4 & 5 & 6 & 7 & 8 & \cdots \\
\operatorname{dim} V & e & e-1 & e-1 & e-2 & e-2 & e-3 & e-3 & e-4 & e-4 & \cdots
\end{array}
$$

Proposition 1.4. A symmetric bilinear map $\phi: E \times E \rightarrow \mathbf{C}$ has rank $\leq 2 p$ if and only if it has an isotropic subspace of dimension $e-p$.

Proof. This follows directly from the table above.

Remarks. (1) As Proposition 1.4 shows, the dimensions of the isotropic subspaces characterize only the bilinear forms of ranks at most an even integer.

(2) Analogously, Proposition 1.4 remains true for a skew-symmetric bilinear map.

\section{A DUALITY CONSTRUCTION}

Let $E$ be a vector space with coordinates $z_{1}, \ldots, z_{e}$. We think of the dual space $E^{*}$, with coordinates $a_{1}, \ldots, a_{e}$, as the space of all hyperplanes in $E$. Then there is a classic duality:

$$
\begin{aligned}
\{\text { points in } E\} & \longleftrightarrow\left\{\text { linear forms on } E^{*}\right\}=\Gamma\left(\mathbf{P}\left(E^{*}\right), \mathscr{O}(1)\right), \\
z=\left(z_{1}, \ldots, z_{e}\right) & \longleftrightarrow z^{*}=\sum_{i=1}^{e} z_{i} a_{i} .
\end{aligned}
$$


Globalizing this simple observation, if now $E$ is a vector bundle over a variety $X$, then a section $s$ of $E$ over $X$ induces a section $s^{*}$ of $\mathscr{O}(1)$ over the projective bundle $\mathbf{P}\left(E^{*}\right)$, where $s^{*}\left(x,\left[e^{*}\right]\right)=e^{*}(s(x))$ for $e^{*} \in E_{x}^{*}$.

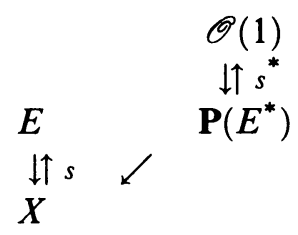

Proposition 2.1. If $E$ is a ranke vector bundle over a variety $X$ and $s$ is a section of $E$ over $X$, then $\mathbf{P}\left(E^{*}\right)-Z\left(s^{*}\right)$ is a $\mathbf{C}^{e-1}$-bundle over $X-Z(s)$, where $Z()$ denotes the zero locus of a section.

Proof. We examine the zero locus of $s^{*}$ on $\mathbf{P}\left(E^{*}\right)$ fiber by fiber. If $s(x)=$ 0 , then $s^{*}$ vanishes on the entire fiber $\mathbf{P}\left(E_{x}^{*}\right)$. At a point $x$ in $X$ where $s(x) \neq 0$, the restriction of $s^{*}$ to the fiber $\mathbf{P}\left(E_{x}^{*}\right)$ vanishes precisely at the point $(x, V)$, where $V$ is the hyperplane in $E_{x}$ defined by $(s(x))^{*}=0$. Therefore, $\mathbf{P}\left(E^{*}\right)-Z\left(s^{*}\right)$ fibers over $X-Z(s)$ with fiber $\mathbf{C}^{e-1}$.

Corollary 2.2. Under the hypothesis of the proposition,

$$
H^{q}\left(\mathbf{P}\left(E^{*}\right)-Z\left(s^{*}\right) ; \mathbf{Z}\right) \simeq H^{q}(X-Z(s) ; \mathbf{Z}) .
$$

The vector bundle $E$ over $X$ is said to be ample if the tautological line bundle $\mathscr{O}(1)$ over $\mathbf{P}\left(E^{*}\right)$ is ample. This implies immediately that $\mathbf{P}\left(E^{*}\right)-$ $Z\left(s^{*}\right)$ is an affine variety.

\section{SYMMETRIC DEGENERACY LOCI}

Given a rank $e$ vector bundle $E$ and a line bundle $L$ over a variety $X$ and a symmetric bundle map $u: E \otimes E \rightarrow L$ over $X$, recall that the rank $r$ degeneracy locus of $u$ is defined to be

$$
D_{r}(u)=\{x \in X \mid \operatorname{rank} u(x) \leq r\} .
$$

For the sake of simplicity we assume in this section that $X$ is smooth and $L$ is trivial. Our goal now is to prove the following theorem.

Theorem 3.1. Let $u: E \otimes E \rightarrow \mathbf{C}$ be a symmetric bundle map over a smooth variety $X$, and $p$ a nonnegative integer. If $\operatorname{Sym}^{2} E^{*}$ is ample and $\operatorname{dim} X-$ $\left(\begin{array}{c}e-2 p+1 \\ 2\end{array}\right) \geq 1$, then $D_{2 p}(u)$ is connected.

By the correspondence between symmetric linear maps and quadratic forms, $u$ may be regarded as a section of the vector bundle $\operatorname{Sym}^{2} E^{*}$ over $X$. Using the characterization of the rank in Proposition 1.4 we can represent the degeneracy locus $D_{2 p}(u)$ as the image of a zero locus, as follows.

Let $\pi: G=G(e-p, E) \rightarrow X$ be the bundle whose fiber at each point $x$ in $X$ is the Grassmannian $G\left(e-p, E_{x}\right)$ of $(e-p)$-dimensional subspaces of the fiber $E_{x}$, and let $S$ be the universal subbundle over $G$. The inclusion map 
$S \hookrightarrow \pi^{*} E$ over $G$ induces the restriction map $\operatorname{Sym}^{2} \pi^{*} E^{*} \rightarrow \operatorname{Sym}^{2} S^{*}$. The section $u: X \rightarrow \operatorname{Sym}^{2} E^{*}$ gives rise naturally to a section $\left(\pi^{*} u\right)(x, V)=u(x)$ of $\pi^{*} \operatorname{Sym}^{2} E^{*}$ over $G(e-p, E)$ and hence by restriction to a section of $\operatorname{Sym}^{2} S^{*}$ over $G$ :

$$
t(x, V)=\left.u(x)\right|_{V}: V \rightarrow V^{*}
$$

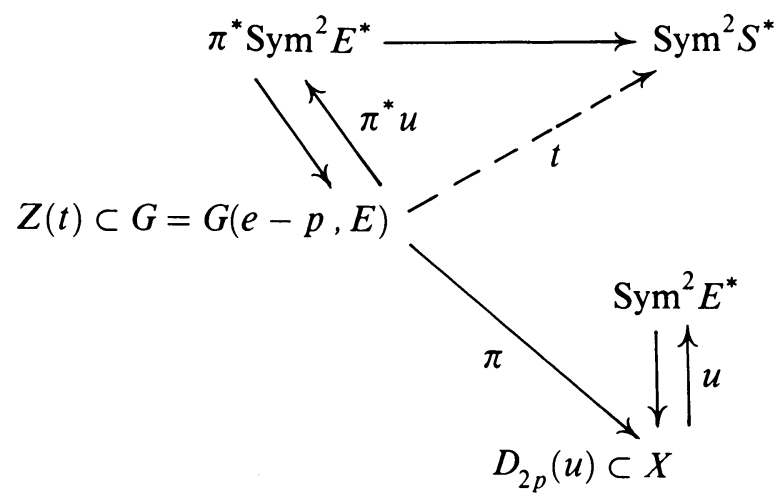

Proposition 3.2. The degeneracy locus $D_{2 p}(u)$ is the image under $\pi$ of the zero locus $Z(t)$.

Proof. This proposition follows from the following series of equivalences:

$x \in D_{2 p}(u)$ iff rank $u(x) \leq 2 p$

iff $u(x)$ has an $(e-p)$-dimensional isotropic subspace $V \subset E_{x}$

iff $t(x, V)=0$

iff $(x, V) \in Z(t)$.

Hence, it suffices to prove the connectedness of the zero locus $Z(t)$.

Lemma 3.3. Let $M$ be a connected compact orientable manifold of (real) dimension $n$ and $A$ a subset of $M$. Then

(i) $A$ is nonempty iff $H^{n}(M-A ; \mathbf{Z})=0$;

(ii) $A$ is connected if $H^{n}(M-A ; \mathbf{Z})=H^{n-1}(M-A ; \mathbf{Z})=0$.

Proof. Both statements are consequences of Lefschetz duality

$$
H^{n-q}(M-A)=H_{q}(M, A)
$$

and the long exact sequence of a pair:

$$
\begin{gathered}
\rightarrow H_{1}(M, A) \rightarrow H_{0}(A) \rightarrow H_{0}(M) \rightarrow H_{0}(M, A) \rightarrow 0 . \\
\mathbf{Z}
\end{gathered}
$$

By this lemma, the connectedness of the locus $Z(t)$ in the Grassmann bundle $G=G(e-p, E)$ follows from the vanishing of the two cohomology groups 
$H^{2 \operatorname{dim} G}(G-Z(t) ; Z)$ and $H^{2 \operatorname{dim} G-1}(G-Z(t) ; Z)$. Instead of computing the cohomology of $G-Z(t)$ directly, we can apply Corollary 2.2 and compute the cohomology of $\mathbf{P}\left(\operatorname{Sym}^{2} S\right)-Z\left(t^{*}\right)$ first. The advantage here is that one can map $\mathbf{P}\left(\operatorname{Sym}^{2} S\right)-Z\left(t^{*}\right)$ to the affine variety $\mathbf{P}\left(\operatorname{Sym}^{2} E\right)-Z\left(u^{*}\right)$, and use the Leray spectral sequence to compare the cohomology of these two spaces. As an affine variety the cohomology of $\mathbf{P}\left(\operatorname{Sym}^{2} E\right)-Z\left(u^{*}\right)$ vanishes above its middle dimension. So the computation of $H^{*}(G-Z(t))$ will be based on the following diagram:

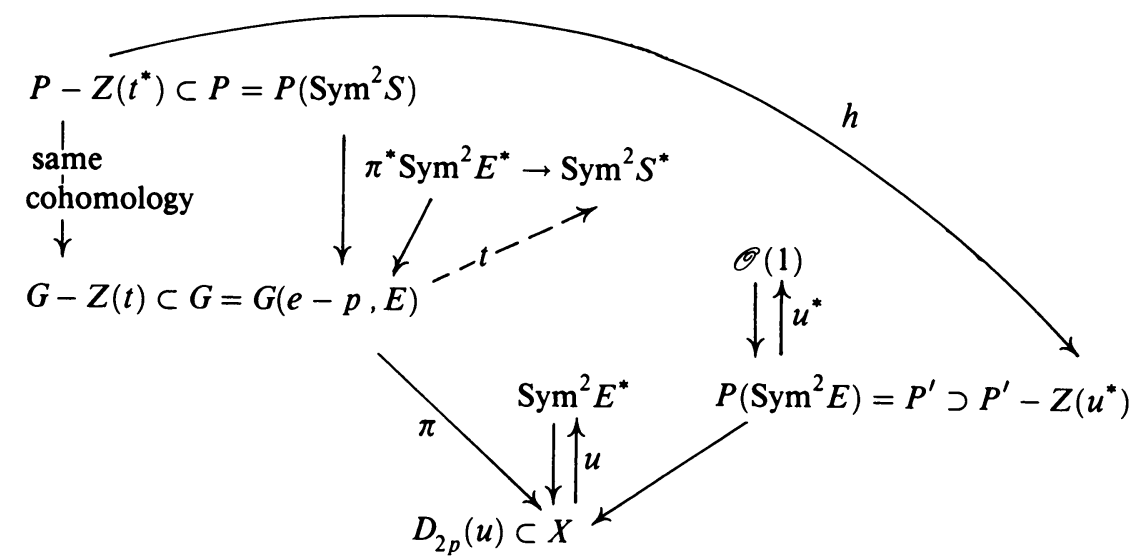

Let $P=\mathbf{P}\left(\operatorname{Sym}^{2} S\right)$ and $P^{\prime}=\mathbf{P}\left(\operatorname{Sym}^{2} E\right)$. In this diagram $P-Z\left(t^{*}\right)$ is an affine-space bundle over $G-Z(t)$ by Proposition 2.1 and therefore has the same cohomology as $G-Z(t)$. By hypothesis $\mathrm{Sym}^{2} E^{*}$ is an ample vector bundle over $X$, so $\mathscr{O}_{P^{\prime}}(1)$ is an ample line bundle over $P^{\prime}$, and $P^{\prime}-Z\left(u^{*}\right)$ is an affine variety. The map $h: \mathbf{P}\left(\operatorname{Sym}^{2} S\right) \rightarrow \mathbf{P}\left(\operatorname{Sym}^{2} E\right)$ is induced from the inclusion of $\operatorname{Sym}^{2} V$ in $\operatorname{Sym}^{2} E_{x}$ :

$$
h\left(x, V \subset E_{x}, \phi \in \operatorname{Sym}^{2} V\right)=\left(x, \phi \in \operatorname{Sym}^{2} E_{x}\right) .
$$

Proposition 3.5. The map $h: P \rightarrow P^{\prime}$ sends $P-Z\left(t^{*}\right)$ to $P^{\prime}-Z\left(u^{*}\right)$.

Proof. By the definition of $t^{*}$,

$$
\begin{gathered}
t^{*}\left(x, V \subset E_{x}, \phi \in \operatorname{Sym}^{2} V\right)=\left(\left.u(x)\right|_{V}\right)^{*}(\phi)=u(x)^{*}(\phi) \\
=u^{*}\left(x, \phi \in \operatorname{Sym}^{2} E_{x}\right)=u^{*}(h(x, V, \phi)) .
\end{gathered}
$$

So if $t^{*}(x, V, \phi) \neq 0$, then $u^{*}(h(x, V, \phi)) \neq 0$ and $h$ sends $P-Z\left(t^{*}\right)$ to $P^{\prime}-Z\left(u^{*}\right)$.

To compute $H^{*}\left(P-Z\left(t^{*}\right)\right)$ we will apply the following cohomology lemma, whose proof is postponed to the next section. Denote by $N$ the set of natural numbers, $\{0,1,2,3, \ldots\}$. 
Lemma 3.6. Let $h: M \rightarrow Y$ be a surjective proper morphism from any variety $M$ to an affine variety $Y$. Suppose there is a strictly increasing function $d: N \rightarrow N$ and a sequence of closed subvarieties

$$
\cdots \subset Y_{k+1} \subset Y_{k} \subset Y_{k-1} \subset \cdots \subset Y_{0}=Y
$$

such that for all $x$ in $Y_{k}-Y_{k+1}$,

$$
d(k)=\operatorname{dim}_{\mathrm{C}} h^{-1}(x)
$$

Define

$$
R=\max _{k \geq 0}\left\{\operatorname{dim}_{\mathbf{C}} Y_{k}+2 d(k)\right\}
$$

Then $H^{q}(M, \mathbf{Z})=0$ for all $q>R$.

To apply this lemma, let $\mathbf{P}\left(D_{k}\left(\operatorname{Sym}^{2} E\right)\right)$ be the subvariety of $\mathbf{P}\left(\operatorname{Sym}^{2} E\right)$ consisting of elements of rank at most $k$, and let

$$
U_{k}=\mathbf{P}\left(D_{k}\left(\operatorname{Sym}^{2} E\right)\right) \cap\left(P^{\prime}-Z\left(u^{*}\right)\right) \text {. }
$$

The image of $h: P-Z\left(t^{*}\right) \rightarrow P^{\prime}-Z\left(u^{*}\right)$ lies in $U_{e-p}$. Define $Y_{k}=U_{e-p-k}$. Then

$$
\cdots \subset Y_{k+1} \subset Y_{k} \subset Y_{k-1} \subset \cdots \subset Y_{0}
$$

and $\operatorname{dim} Y_{k}=\operatorname{dim} P^{\prime}-\left(\begin{array}{c}p+k+1 \\ 2\end{array}\right)$. For $\left(x, \phi \in \operatorname{Sym}^{2} E_{x}\right) \in Y_{k}-Y_{k+1}, \phi$ may be viewed as a symmetric map $\phi: E_{x}^{*} \rightarrow E_{x}$ of rank exactly $e-p-k$, so that $\operatorname{im} \phi$ is an $(e-p-k)$-dimensional subspace of $E_{x}$. Thus,

$$
\begin{aligned}
h^{-1}(x, \phi) & \simeq\left\{V \in G\left(e-p, E_{x}\right) \mid \operatorname{im} \phi \subset V \subset E_{x}\right\} \\
& \simeq G\left((e-p)-(e-p-k), E_{x} / \operatorname{im} \phi\right) \\
& \simeq G(k, p+k) .
\end{aligned}
$$

Therefore, for $(x, \phi) \in Y_{k}-Y_{k+1}, \operatorname{dim}_{\mathrm{C}} h^{-1}(x, \phi)=p k$. In the cohomology lemma (3.6) set $d(k)=p k$. Then

$$
\begin{aligned}
R & =\max _{k \geq 0}\left\{\operatorname{dim} Y_{k}+2 p k\right\} \\
& =\max _{k \geq 0}\left\{\operatorname{dim} P^{\prime}-\left(\begin{array}{c}
p+k+1 \\
2
\end{array}\right)+2 p k\right\} \\
& =\max _{k \geq 0}\left\{\operatorname{dim} P^{\prime}-p-\frac{(k-p+1)(k-p)}{2}\right\} .
\end{aligned}
$$

For integer values of $p$ and $k$, the minimum of $(k-p+1)(k-p) / 2$ is 0 , when $k=p$ or $p-1$. Therefore,

$$
R=\operatorname{dim} P^{\prime}-p=\operatorname{dim} X+\left(\begin{array}{c}
e+1 \\
2
\end{array}\right)-1-p .
$$

By Lemma 3.6,

$$
H^{q}\left(P-Z\left(t^{*}\right) ; \mathbf{Z}\right)=0 \text { for } q \geq \operatorname{dim} X+\left(\begin{array}{c}
e+1 \\
2
\end{array}\right)-p .
$$


A straightforward computation shows that

$$
\operatorname{dim} X \geq\left(\begin{array}{c}
e-2 p+1 \\
2
\end{array}\right)+1 \Leftrightarrow 2 \operatorname{dim} G-1 \geq \operatorname{dim} X+\left(\begin{array}{c}
e+1 \\
2
\end{array}\right)-p
$$

Therefore, if $\operatorname{dim} X \geq\left(\begin{array}{c}e-2 p+1 \\ 2\end{array}\right)+1$, then

$$
H^{2 \operatorname{dim} G}(G-Z(t) ; \mathbf{Z})=H^{2 \operatorname{dim} G-1}(G-Z(t) ; \mathbf{Z})=0 \text {. }
$$

By Proposition 3.2 and the remark following Lemma 3.3, $Z(t)$ and hence $D_{2 p}(u)$ is connected.

\section{Proof of THE COHOMOLOGY LEMMA}

We prove here Lemma 3.6, which allows one to compare the cohomology of a variety $M$ with the cohomology of an affine image $Y$ of $M$. For technical reasons that will be apparent shortly, we extend the definition of the fiberdimension function $d$ to $\{-1,0,1,2, \ldots\}$ by defining $d(-1)=-1$. This makes sense, for the fibers over the empty set $Y_{-1}-Y_{0}$ are of course the empty sets. In the notations of Lemma 3.6, applying the Leray spectral sequence to the surjective map $h: M \rightarrow Y$, we find that $H^{j}\left(Y, R^{i} h_{*} \mathbf{Z}\right)$ abuts to $H^{i+j}(M, \mathbf{Z})$. So it suffices to prove that $H^{j}\left(Y, R^{i} h_{*} \mathrm{Z}\right)=0$ for all $i+j>R$.

Claim. Whenever there is a $k \in N$ such that

$$
i>2 d(k-1) \text { and } j>\operatorname{dim} Y_{k},
$$

then $H^{j}\left(Y, R^{i} h_{*} \mathbf{Z}\right)=0$.

Proof. Suppose $i>2 d(k-1)$. For dimension reasons, $R^{i} h_{*} \mathbf{Z}$ vanishes on $Y-Y_{k}$. Thus, $R^{i} h_{*} \mathrm{Z}$ is concentrated on $Y_{k}$ and

$$
H^{j}\left(Y, R^{i} h_{*} \mathbf{Z}\right)=H^{j}\left(Y_{k}, R^{i} h_{*} \mathbf{Z}\right)
$$

As a direct image of a constructible sheaf, $R^{i} h_{*} \mathrm{Z}$ is again constructible on $Y_{k}$. By a standard theorem, the cohomology of a constructible sheaf on an affine variety vanishes above the dimension of the variety. So $H^{j}\left(Y_{k}, R^{i} h_{*} \mathrm{Z}\right)=0$ for $j>\operatorname{dim} Y_{k}$. This concludes the proof of the claim.

Since $d:\{-1,0,1,2, \ldots\} \rightarrow\{-1,0,1,2, \ldots\}$ is a strictly increasing function, given any nonnegative integer $i$ we can choose $k$ such that

$$
2 d(k) \geq i>2 d(k-1) .
$$

(This inequality is the reason the domain and the image of the function $d$ must contain -1 , since $i$ may be 0 .) If $i+j>R$, then

$$
j>R-i \geq R-2 d(k) \geq \operatorname{dim} Y_{k},
$$

where the last inequality follows from the definition of $R$. By (4.1),

$$
H^{j}\left(Y_{k}, R^{i} h_{*} \mathbf{Z}\right)=0 \text { for all } i+j>R \text {. }
$$




\section{Generalizations}

In $\S 3$ we proved the connectedness conjecture $(0.2)$ for an even-rank symmetric degeneracy locus under the assumptions that the ambient space $X$ is smooth and that the line bundle $L$ is trivial. It is not difficult to remove both restrictions.

In the proof of $\S 3$ the smoothness assumption was needed only to invoke Lefschetz duality and to conclude that if $H^{q}(G-Z(t) ; \mathbf{Z})=0$ for $q=2 \operatorname{dim} G$, $2 \operatorname{dim} G-1$, then $H_{q}(G, Z(t) ; Z)=0$ for $q=0,1$. Thus, to generalize from a smooth variety to an arbitrary irreducible variety $X$, what is needed is a substitute for Lefschetz duality. This is provided by an argument in [FL1], which in fact goes through verbatim in the present situation. Their idea is first to show that one may assume $X$ normal. For if $\nu: \widetilde{X} \rightarrow X$ is the normalization of the irreducible variety $X$, then $\widetilde{X}$ is also irreducible. Furthermore, the section $u$ of $\operatorname{Sym}^{2} E^{*}$ over $X$ pulls back to a section $\nu^{*} u$ of $\operatorname{Sym}^{2} E^{*}$ over $\tilde{X}$, and $D_{r}(u)$ is the image of $D_{r}\left(\nu^{*} u\right)$ under $\nu$. Since the pullback of an ample vector bundle under a finite morphism is again ample, $\nu^{*} \operatorname{Sym}^{2} E^{*}$ is ample. So it suffices to prove the connectedness of $D_{r}\left(\nu^{*} u\right)$. Replacing $X$ by $\widetilde{X}$, we may assume $X$ normal. Then the Grassmann bundle $G$ over $X$ is also normal, and the following lemma applies.

Lemma [FL1, Lemma 1.3]. Let $G$ be a normal projective variety of dimension $m$ and let $Z \subset G$ be a closed algebraic subset. Then there is an injection

$$
H^{1}(G, Z ; \mathbf{Q}) \hookrightarrow H_{2 m-1}(G-Z ; \mathbf{Z}) .
$$

By the universal coefficient theorem, $H^{q}(G-Z(t) ; \mathbf{Z})=0$ for $q=2 \operatorname{dim} G$, $2 \operatorname{dim} G-1$ implies that $H_{2 \operatorname{dim} G-1}(G-Z(t) ; \mathbf{Q})=0$ [M, Corollary 56.4 and Theorem 53.5]. Hence, $H^{1}(G, Z(t) ; \mathbf{Z})=0$ and $Z(t)$ is connected. This concludes the proof when $X$ is an irreducible variety.

Finally, suppose $L$ is an arbitrary line bundle. If $L$ happens to be the square of a line bundle, say $L \simeq K \otimes K$, then $\left(\operatorname{Sym}^{2} E^{*}\right) \otimes L \simeq \operatorname{Sym}^{2}\left(E^{*} \otimes K\right)$ and Conjecture 0.2 for even ranks would follow immediately from Theorem 3.1 (but with $X$ an irreducible instead of a smooth variety). The following lemma of Bloch and Gieseker effectively allows one to assume that every bundle is a square.

Lemma 5.1 [BG, Lemma 2.1]. Let $L$ be a line bundle on a projective variety $X$ and $d$ a positive integer. Then there exists a projective variety $Y$, a finite surjective morphism $f: Y \rightarrow X$, and a line bundle $K$ on $Y$ such that $f^{*} L \simeq$ $K^{\otimes d}$.

Proof. See [BG, p. 114 or FL2, Lemma 1.1].

Returning to the proof of Conjecture 0.2 for even ranks, we take $d=2$ in Lemma 5.1. Then

$$
\left.f^{*}\left(\left(\operatorname{Sym}^{2} E^{*}\right) \otimes L\right)\right) \simeq\left(f^{*} \operatorname{Sym}^{2} E^{*}\right) \otimes K^{2} \simeq \operatorname{Sym}^{2}\left(f^{*} E^{*} \otimes K\right) .
$$


The degeneracy locus $D_{r}\left(f^{*} u\right)$ in $Y$ maps surjectively onto $D_{r}(u)$ in $X$. Since the pullback of an ample vector bundle under a finite morphism is again ample, we conclude by Theorem 3.1 (again with $X$ irreducible instead of smooth) that $D_{r}\left(f^{*} u\right)$ is connected. Therefore, $D_{r}(u)$ is also connected.

\section{SKEW-SYMMETRIC DEGENERACY LOCI}

By the argument of $\S 5$, Conjecture 0.3 can also be reduced to the case where $X$ is a smooth variety and $L$ is a trivial line bundle. To prove Conjecture 0.3 under these assumptions, replace $\operatorname{Sym}^{2}$ by $\Lambda^{2}$ in Diagram 3.4. This gives a map $h: P-Z\left(t^{*}\right) \rightarrow P^{\prime}-Z\left(u^{*}\right)$, where $P=\mathbf{P}\left(\bigwedge^{2} S\right)$ and $P^{\prime}=\mathbf{P}\left(\bigwedge^{2} E\right)$. Let $U_{k}=\mathbf{P}\left(D_{k}\left(\bigwedge^{2} E\right)\right) \cap P^{\prime}-Z\left(u^{*}\right)$. The proof proceeds as in the even-rank symmetric case, except now because the rank of a skew-symmetric matrix is always even, the image of $h$ lies in $U_{e-p}$ or $U_{e-p-1}$ depending on whether $e-p$ is even or odd, and the rank drops by two at a time. To combine the two cases in one proof, let

$$
l= \begin{cases}2 k, & \text { if } e-p \text { is even, } \\ 2 k+1, & \text { if } e-p \text { is odd }\end{cases}
$$

and define $Y_{k}=U_{e-p-l}$. Then the image of $h$ lies in $Y_{0}$,

$$
\operatorname{dim} Y_{k}=\operatorname{dim} P^{\prime}-\left(\begin{array}{c}
p+l \\
2
\end{array}\right)
$$

and

$$
\cdots \subset Y_{k+1} \subset Y_{k} \subset Y_{k-1} \subset \cdots \subset Y_{0} .
$$

If $\left(x, \phi \in \bigwedge^{2} E_{x}\right) \in Y_{k}-Y_{k+1}$, then

$$
h^{-1}(x, \phi) \simeq G(l, p+l) \text { and } \operatorname{dim}_{\mathrm{C}} h^{-1}(x, \phi)=p l .
$$

In the cohomology lemma (3.6),

$$
\begin{aligned}
R & =\max _{k \geq 0}\left\{\operatorname{dim} P^{\prime}-\left(\begin{array}{c}
p+l \\
2
\end{array}\right)+2 p l\right\} \\
& =\max _{k \geq 0}\left\{\operatorname{dim} P^{\prime}+p-\frac{(p-l+1)(p-l)}{2}\right\} \\
& =\operatorname{dim} P^{\prime}+p=\operatorname{dim} X+\left(\begin{array}{l}
e \\
2
\end{array}\right)-1+p .
\end{aligned}
$$

By Lemma (3.6),

$$
H^{q}\left(P-Z\left(t^{*}\right) ; \mathbf{Z}\right)=0 \quad \text { for } q \geq \operatorname{dim} X+\left(\begin{array}{l}
e \\
2
\end{array}\right)+p .
$$

Again one checks easily that

$$
\operatorname{dim} X \geq\left(\begin{array}{c}
e-2 p \\
2
\end{array}\right)+1 \Leftrightarrow 2 \operatorname{dim} G-1 \geq \operatorname{dim} X+\left(\begin{array}{l}
e \\
2
\end{array}\right)+p .
$$

So under the hypotheses of Conjecture 0.3,

$$
H^{2 \operatorname{dim} G}(G-Z(t) ; \mathbf{Z})=H^{2 \operatorname{dim} G-1}(G-Z(t) ; \mathbf{Z})=0
$$

and the connectedness of $D_{2 p}(u)$ follows as before. 


\section{REFERENCES}

[ACGH] E. Arbarello, M. Cornalba, P. Griffiths, and J. Harris, Geometry of algebraic curves, Springer-Verlag, New York, 1985.

[BG] S. Bloch and D. Gieseker, The positivity of the Chern classes of an ample vector bundle, Invent. Math. 12 (1971), 112-117.

[FL1] W. Fulton and R. Lazarsfeld, On the connectedness of degeneracy loci and special divisors, Acta Math. 146 (1981), 271-283.

[FL2] W. Fulton and R. Lazarsfeld, Positive polynomials for ample vector bundles, Ann. of Math. 118 (1983), 35-60.

[HT] J. Harris and L. W. Tu, On symmetric and skew-symmetric determinantal varieties, Topology 23 (1984), 71-84.

[HT2] J. Harris and L. W. Tu, The connectedness of symmetric degeneracy loci: odd ranks, Proc. Minisemester on Commutative Algebra and Algebraic Geometry (Warsaw, 1988) (to appear).

[L] S. Lang, Algebra, Addison-Wesley, Menlo Park, Calif., 1984.

[M] J. R. Munkres, Elements of algebraic topology, Addison-Wesley, Menlo Park, Calif., 1984.

[P] P. Pragacz, Cycles of isotropic subspaces and formulas for symmetric degeneracy loci, Proc. Minisemester on Commutative Algebra and Algebraic Geometry (Warsaw, 1988) (to appear).

[S] A. Sommese, Submanifolds of abelian varieties, Math. Ann. 233 (1978), 229-256.

[T] L. W. Tu, Degeneracy loci, Proc. Internat. Conf. Algebraic Geometry (Berlin, 1985), Teubner Verlagsgesellschaft, Leipzig, 1986, pp. 296-305.

[T2] L. W. Tu, The connectedness of degeneracy loci, Proc. Minisemester on Commutative Algebra and Algebraic Geometry (Warsaw, 1988) (to appear).

Department of Mathematics, Tufts University, Medford, Massachusetts 02155 